\title{
Nasoangiofibroma juvenil: \\ Nuestra experiencia en los últimos 10 años y revisión de la literatura
}

\author{
Juvenile Nasopharyngeal Angiofibroma: \\ Our experience in the last $\mathbf{1 0}$ years and review of the literature
}

Carlos Mena $\mathrm{C}^{1}$, Gilda Bogado R², Clavigo Klassen $Z^{2}$.

\section{RESUMEN}

Introducción: El nasoangiofibroma juvenil es un tumor benigno con comportamiento de agresividad local, dadas las importantes epistaxis que provoca, la frecuente recidiva local y la complejidad terapéutica que se presenta ante esta patología; se presenta exclusivamente en hombres adolescentes y corresponde al 0,5\%-0,05\% de todos los tumores de cabeza y cuello.

Objetivo: Determinar el manejo y las características del paciente con el diagnóstico de nasoagiofibroma juvenil que acude a nuestro servicio. Evaluar necesidad de transfusión sanguínea. Exponer las complicaciones encontradas.

Material y método: Se realizó un trabajo retrospectivo observacional de corte transversal de pacientes con diagnóstico de nasoangiofibroma juvenil (NAJ), confirmado por estudios histológicos en la Cátedra de Otorrinolaringología de la Facultad de Ciencias Médicas de la Universidad Nacional de Asunción, desde enero de 1998 hasta septiembre de 2008.

Resultados: Con un total de 45, de los cuales 44 recibieron tratamiento quirúrgico. El rango de edad estaba comprendido entre 7 a 34 años, con una mediana de 16 años y un promedio de 17 años, todos de sexo masculino. Se presentaron con mayor frecuencia en estadio Fisch II, rango de evolución sintomática de 1 a 60 meses, con una mediana de 7 meses de evolución de epistaxis recurrente, obstrucción nasal y rinorrea. La mayoría de grupo sanguíneo $0 \mathrm{Rh}+$, provenientes del Departamento Central, recibiendo como tratamiento la resección endoscópica. Siete pacientes presentaron recidivas, con una mediana de 7 meses desde el tratamiento. En 58,8\% de los procedimientos quirúrgicos fue necesaria la transfusión de derivados sanguíneos.

Conclusión: Los pacientes que acuden al servicio con el diagnóstico de nasoangiofibroma juvenil, son sometidos a tratamiento quirúrgico en la totalidad de los casos. En la mayoría de los casos se requirió algún tipo de transfusión sanguínea. No hubo complicaciones por el procedimiento quirúrgico.

Palabras clave: Nasoangiofibroma juvenil, epistaxis recurrente, degloving mediofacial modificado.

1 Médico Otorrinolaringólogo del Hospital de Clínicas, Universidad Nacional de Asunción-Paraguay.

2 Estudiante de la Universidad Nacional de Asunción-Paraguay. 


\section{ABSTRACT}

Introduction: The juvenile Nasopharyngeal Angiofibroma is a benign tumor with behavior of local aggressiveness, given the important epistaxis that it provokes, the frequent local recidiva and the therapeutic complexity that one presents before this pathology; he appears exclusively in teen men and corresponds to 0,5\%-0,05\% of all the tumors of head and neck.

Aim: To determine the managing and the characteristics of the patient with juvenile Nasopharyngeal Angiofibroma diagnosis that comes to our service. To evaluate need of blood transfusion. To expose the opposing complications.

Material and method: There realized a retrospective work observacional of patients' transverse court (cut) with diagnosis of juvenile Nasopharyngeal Angiofibroma (NAJ), confirmed by histological studies in the Service of Otolaryngology of the Asunción's National University, from January, 1998 until September, 2008.

Results: With a whole of 45, of which 44 received surgical treatment. The range of age was understood (included) between (among) 7 to 34 years, with a median of 16 years and an average of 17 years, all of masculine sex. Appellants presented with major frequency in estadío Fisch II, range of symptomatic evolution from 1 to 60 months, with a median of 7 months of evolution of epistaxis, nasal obstruction and rinorrea. The majority of blood group $0 \mathrm{Rh}+$, from the Central department, receiving as treatment the resection endoscopic. 7 patients presented recidivas, with a median of 7 months from the treatment. In $58,8 \%$ of the surgical procedures there was necessary the transfusion of blood derivatives.

Conclusion: The patients who come to the service with juvenile nasopharyngeal angiofibroma diagnosis, are submitted to surgical treatment in the totality of the cases. In most cases there was needed some type of blood transfusion. There were no complications for the surgical procedure.

Key words: Juvenile Nasopharyngeal Angiofibroma, Epistaxis appellants, Midface Degloving Modified

\section{INTRODUCCIÓN}

El nasoangiofibroma juvenil es un tumor histológica y biológicamente benigno con comportamiento de agresividad local, dadas las importantes epistaxis que provoca, la frecuente recidiva local y la complejidad terapéutica que se presenta ante esta patología ${ }^{1-4}$.

Se presenta casi exclusivamente en hombres adolescentes y corresponde al $0,5 \%-0,05 \%$ de todos los tumores de cabeza y cuello, variando esta cifra en algunos países asiáticos y africanos $^{1,4,5}$.

El tumor invade la fosa infratemporal, el etmoides, la región orbitaria y el esfenoides en $90 \%$ de los casos. La fosa pterigopalatina en $76 \%$. La incidencia de invasión cerebral varía de 4,3\% a $11 \%$ pudiendo llegar incluso hasta $26 \%{ }^{4}$.

El diagnóstico presuntivo es de capital importancia, basándose principalmente en el interrogato- rio y el examen clínico. La biopsia solamente se aconseja en casos de duda diagnóstica ${ }^{3}$. La tomografía computarizada y la resonancia magnética permiten establecer claramente la extensión tumoral, su patrón de diseminación y la planeación de abordajes quirúrgicos. A partir de éstas, han surgido múltiples clasificaciones como la de Sessions, Fisch y Chandler ${ }^{1,3,4,6,7}$.

En cuanto al tratamiento, se menciona el uso de hormonas (estrógeno, testosterona) radioterapia, quimioterapia a base de doxorrubicina y decarbacina, y recientemente el uso de embolización. La mayoría de los autores coinciden en que la cirugía constituye el tratamiento de elección y la vía depende del estadio clínico y el estado general del paciente $e^{3,4,7-10}$.

El objetivo de este trabajo es determinar el manejo y las características del paciente con el diagnóstico de nasoangiofibroma juvenil, que acude al servicio de ORL del Hospital de Clínicas de la 
FCM-UNA, Asunción, Paraguay durante el período comprendido entre enero de 1998 hasta septiembre de 2008. Aquí se evaluará la necesidad de transfusión sanguínea en el perioperatorio de estos pacientes; se expondrán las complicaciones encontradas en estas cirugías y en el posoperatorio y se describirá el seguimiento de los pacientes sometidos a cirugía y el manejo de las recidivas.

\section{MATERIAL Y MÉTODO}

Trabajo descriptivo, observacional retrospectivo de corte transverso, con componente analítico. Se estudiaron historias clínicas de 45 pacientes con nasoangiofibroma juvenil internados en la Cátedra de Otorrinolaringología de la Facultad de Ciencias Médicas de la Universidad Nacional de Asunción entre enero de 1998 y septiembre de 2008. Se incluyeron todos los que fueron sometidos a extirpación quirúrgica en el servicio y contaban con confirmación histológica. Se excluyeron todos los que no cumplían los requisitos arriba mencionados. Se estudiaron las siguientes variables: edad, sexo, procedencia, grupo sanguíneo, síntomas y su evolución, estadio, tratamiento, complicaciones inmediatas y tardías, recidivas, progresión de la enfermedad, comorbilidades.

Según los hallazgos clínicos, radiológicos y quirúrgicos, todos los casos fueron clasificados de acuerdo a los estadios propuestos por Fisch en 1983: (estadios por tomografía axial computarizada. Fotos obtenidas de tomografías de pacientes con nasoangiofibroma juvenil del Servicio de ORL del Hospital de Clínicas de la FCMUNA).

Estadio I: Tumor limitado a la nasofaringe y cavidad nasal sin destrucción ósea.

Estadio II: Tumor invade la fosa pterigomaxilar, antro maxilar, seno etmoidal y esfenoidal con destrucción ósea.
Estadio III: Tumor invade la fosa infratemporal, órbita y región paraselar permaneciendo lateral al seno cavernoso.

Estadio IV: Tumor con invasión masiva al seno cavernoso, región del quiasma óptico o fosa pituitaria.

Los datos obtenidos fueron almacenados en una tabla de Microsoft Office Excel, donde fueron analizados posteriormente.

\section{RESULTADOS}

De los 45 pacientes diagnosticados de nasoangiofibroma juvenil recibieron tratamiento quirúrgico 44. Todos ellos son del sexo masculino en edades comprendidas entre 7 y 34 años, con una mediana de 16 años. La siguiente tabla ilustra los grupos sanguíneos de los pacientes (Tabla 1).

Todos los pacientes provenían de la región oriental del Paraguay. Estos consultaron por primera vez en un promedio de 10,2 meses luego del inicio de los síntomas, con una mediana de 7 meses. La persona que consultó más precozmente lo hizo en 1 mes y la consulta más tardía fue a los 60 meses del inicio de los síntomas. La epistaxis recurrente fue el motivo de consulta de la totalidad de los pacientes, a ella acompañan obstrucción nasal y rinorrea. Catorce $(31,8 \%)$ pacientes presentaron además otros síntomas tales como anosmia, hiposmia, hipoacusia, cefalea frontal, abombamiento de mejilla y trastornos visuales. La Figura 1 ilustra el estadio de los pacientes según la clasificación de Fisch.

Los tratamientos instaurados fueron el Degloving Mediofacial Modificado (DMF) en 45\% de los pacientes, y resección por endoscopía en $55 \%$ de los pacientes, en tres de éstos se utilizó un abordaje transpalatino. Pacientes en estadío Fisch IV fueron tratados exclusivamente con DMF. Com-

Tabla 1. Se detalla la cantidad de pacientes en los diferentes grupos sanguíneos encontrados

\begin{tabular}{|cc|}
\hline Grupo sanguíneo & Cantidad \\
\hline $0 \mathrm{Rh}+$ & 25 \\
$\mathrm{ARh}+$ & 15 \\
$\mathrm{BRh}+$ & 4 \\
\hline
\end{tabular}




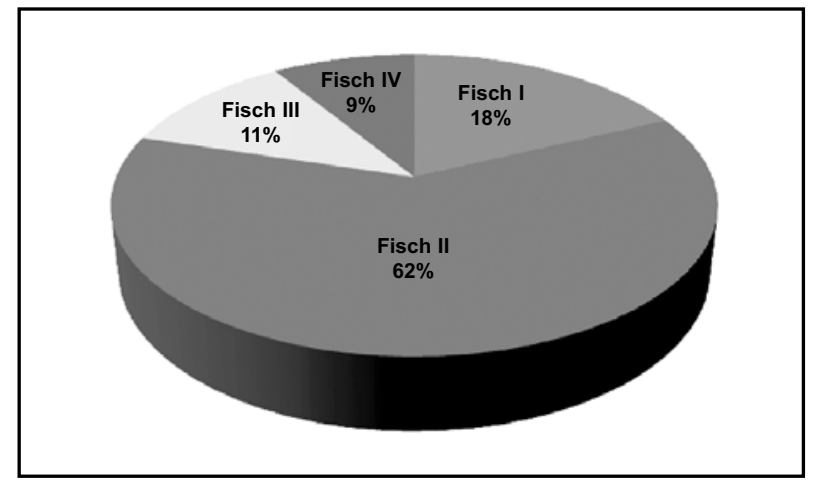

Figura 1. Distribución en porcentajes de los pacientes de nuestro estudio según el estadio de Fisch al que pertenecieron.

plicaciones ocurrieron en el $25 \%$ de los casos, entre ellas se mencionan la hemorragia, diabetes insípida de origen central, uno de ellos presentó convulsiones generalizadas, delirium agudo y paro cardiorrespiratorio. Un paciente falleció por causas no especificadas en la historia clínica. Las recidivas se presentaron en 7 (16\%) pacientes, algunos de los cuales (3 pacientes) la presentaron en más de una ocasión. Las recidivas ocurrieron en un período de tiempo de 5 a 66 meses después del tratamiento inicial, con una mediana de 7 meses. Del total de pacientes, 14 nunca volvieron para control luego del alta y de entre los que sí lo hicieron 25 pacientes tuvieron un seguimiento menor 0 igual a seis meses. La mediana para el seguimiento fue de 6,5 meses con un rango de 0 a 96 meses poscirugía.

En el servicio durante el período mencionado se realizaron 51 cirugías por nasoangiofibroma juvenil, de ellas 9 eran para tratamiento de recidivas y 42 por el tumor primario. En dos casos se realizó embolización prequirúrgica y en un caso se requirió ligadura de la arteria maxilar interna por epistaxis masiva. En treinta de estos procedimientos $(59 \%)$ se requirió algún tipo de transfusión, ya sean glóbulos rojos concentrados $(73 \%)$, sangre total $(26 \%)$, plasma fresco congelado $(20 \%) 0$ plaquetas $(6 \%)$ separados 0 en diversas combinaciones. El promedio de glóbulos rojos concentrados (GRC) requeridos fue de 2,5 volúmenes (400 cc/volumen), el promedio para la ST fue de 2,25 volúmenes (450 cc/volumen) y el de PFC fue de 7,1 volúmenes (200 cc/volumen). En cuanto a las diferencias entre el dosaje de hemoglobina pre y poscirugía, encontramos que la mediana de la prequirúrgica era de $14,5 \mathrm{mg} / \mathrm{dl}$, con un rango de $8,5-15,5 \mathrm{mg} / \mathrm{dl}$ y que la posquirúrgica variaba entre 6,5 y $14,4 \mathrm{mg} / \mathrm{dl}$ con una mediana de $11,5 \mathrm{mg} / \mathrm{dl}$. Debemos resaltar que en 11 casos no se contaba con el dosaje de hemoglobina posoperatoria, pero que todos contaban con el dosaje preoperatorio.

\section{DISCUSIÓN}

Aunque encontramos un rango de edad mayor del que se maneja en estudios de México, Nicaragua y otros países en los cuales el rango varía entre 14 a 24 años nuestra mediana de la edad de presentación sí es muy similar, 17 años. Todos coinciden que los pacientes son del sexo masculino ${ }^{3,9}$.

Así como en un trabajo realizado en México, la mayoría de nuestros pacientes presentaba el grupo sanguíneo $0 \mathrm{Rh}+$, pero consideramos, que esto se debe a que la mayor parte de la población tanto a nivel mundial como en nuestro país pertenece a ese grupo, y no a un factor genético que relacione ambas variables entre síl.

El principal síntoma que motivó la consulta fue la epistaxis, seguida por la obstrucción nasal y la rinorrea, al igual que en la casuística de un estudio colombiano; en cambio en estudios realizados en EE.UU. Io que motivaba al paciente a consultar era la obstrucción y en segundo lugar la epistaxis. Nuestros pacientes consultaron una mediana de 7 meses luego del inicio de los síntomas, en estudios norteamericanos los pacientes consultan, en su mayoría, antes de los 6 
meses del inicio de los síntomas. Al igual que estudios realizados en Nicaragua, Estados Unidos, Colombia gran parte de nuestros pacientes se hallaba en el estadío $\mathrm{II}^{3,10-12}$.

En nuestra casuística, el único tratamiento instaurado a los pacientes sin importar la edad o el estadío fue el quirúrgico; en Inglaterra se reporta el caso de un paciente de 26 años con un estadio Fisch IV que fue exitosamente tratado con radioterapia radical. También se reportan casos de pacientes en estadio Fisch IV, inoperables, que fueron tratados con radioterapia en Los Ángeles, EE.UU. $6,7,13$.

Nuestros pacientes, sólo recibieron transfusión aquellos que presentaban una hemorragia importante 0 alteración del estado hemodinámico; en Innsbruck, Austria, todos los pacientes recibían transfusión en el preoperatorio inmediato en forma profiláctica ${ }^{11}$.

Las complicaciones mencionadas en la literatura internacional como secuelas cicatriciales faciales, nasales o bucales no fueron encontradas en nuestro estudio, sí las hemorragias, que sólo fueron consideradas una complicación cuando causaban gran desequilibrio hemodinámico al paciente $e^{3,7,9,10}$.

El porcentaje de recidivas encontrado por nosotros se corresponde con los datos obtenidos por austriacos, norteamericanos y latinoamericanos $3,6,7,9-11$.

La mortalidad que encontramos se ajusta a la esperada según la literatura consultada, aunque en estudios de EE.UU., Nicaragua no tuvieron fallecidos. Un trabajo realizado en Colombia sí presentó una mortalidad posoperatoria similar ${ }^{3,10}$.

\section{CONCLUSIONES}

Los pacientes que acuden al servicio son sometidos a tratamiento quirúrgico en la totalidad de los casos, con extirpación total del tumor, sin embolización previa en la mayoría de los casos por motivos económicos. En más de la mitad de los casos se requirió algún tipo de transfusión sanguínea, e incluso grandes volúmenes de componentes sanguíneos. A excepción de las hemorragias, no hubo complicaciones por el procedimiento quirúrgico. Un paciente falleció por causas no especificadas en la historia clínica. Todos los pacientes que presentaron recidiva en el posoperatorio fueron sometidos a un segundo tiempo quirúrgico de forma inmediata, luego del diagnóstico de la recidiva. La mayor parte de los pacientes recibió un seguimiento menor a 6 meses porque los mismos no acuden a los controles.

\section{Agradecimientos}

Agradecemos al Prof. Dr. José Luis Roig Ocampos, Jefe del Servicio de ORL, por permitirnos usar el archivo del Servicio para la realización de este trabajo.

\section{BIBLIOGRAFÍA}

1. Gaeta Mendoza M. Nasoangiofibroma juvenil frecuencia. Incidencia de acuerdo a grupo sanguíneo. An ORL Méx 2005; 50: 17-21.

2. WYLIE JP, SLEVIN NJ AND JOHNSON RJ. Intracranial juvenile nasopharyngeal angiofibroma. Clinical Oncology 1998; 10: 330-3.

3. Sánchez de Guzmán G. Experiencia en el manejo de nasoangiofibroma juvenil en el Instituto Nacional de Cancerología. Acta de Otorrinolaringologia \& Cirugia de Cabeza y Cuello. Volumen 27, Número 3, septiembre de 1999.

4. Sociedade Brasileira de Otorrinolaringologia. Tratado de otorrinolaringología. Volumen 3. Editorial Roca LTDA. $1^{\circ}$ edición. Cap. 2003; 70: 701-11.

5. Dillard DG, Cohen C, Muller S, Del Gaudio J, Reichman 0, Parrish B, Rackley D, Gal aA. Immunolocalization of Activated Transforming Growth Factor 1 in Juvenile Nasopharyngeal Angiofibrom. Arch Otolaryngol Head Neck Surg 2000; 126: 723-5.

6. McAfee WJ, Morris CG, Amdur RJ, Werning JW, Mendenhall WM. Definitive Radiotherapy for Juvenile Nasopharyngeal Angiofibroma. American Journal of Clinical Oncology 2006; 29: 168-70.

7. Pryor SG, Moore EJ, Kasperbauer JL. Endoscopic versus Traditional Approaches for Excision of Juvenile NasopharyngealAngiofibroma. Laryngoscope 2005; 115: 1201-7.

8. Bales C, Kotapka M, Loevner LA, Al-Rawi M, Weinstein G, Hurst R, Weber RS. Craniofacial Resection of Advanced Juvenile Nasopharyngeal Angiofibroma. Arch Otolaryngol Head Neck Surg 2002; 128: 1071-8.

9. Muñoz del Castillo F, Jurado Ramos A, Bravo-Rodríguez F, Delgado Acosta F, López Vlllarejo P. Cirugía 
endoscópica del angiofibroma nasofaríngeo. Acta Otorrinolaringológica Española 2004; 55, $\mathrm{n}^{\circ} 8$.

10. Gullane PJ, Davison J, O'Dwyer T, Forte V. Juvenile Angiofibroma: A Review of the Literature and Case Series Report. Laryngoscope 1992; 102: 928-33.

11. Scholtz AW, Appenroth E, Kammen-Jolly K, Scholtz LU, Thumfart WF. Juvenile Nasopharyngeal Angiofibroma: Management and Therapy. Laryngoscope 2001; 111: 681-7.
12. Lee JT, Chen P, Safa A, Juillard G, Calcaterra TC. The Role of Radiation in the Treatment of Advanced Juvenile Angiofibroma. Laryngoscope 2002; 112: 1213-20.

13. Zhang M, Garvis W, Linder T, Fisch U. Update on the Infratemporal Fossa Approaches to Nasopharyngeal Angiofibroma. The Laryngoscope 1998; 108 (11, Part 1): 1717-23. 